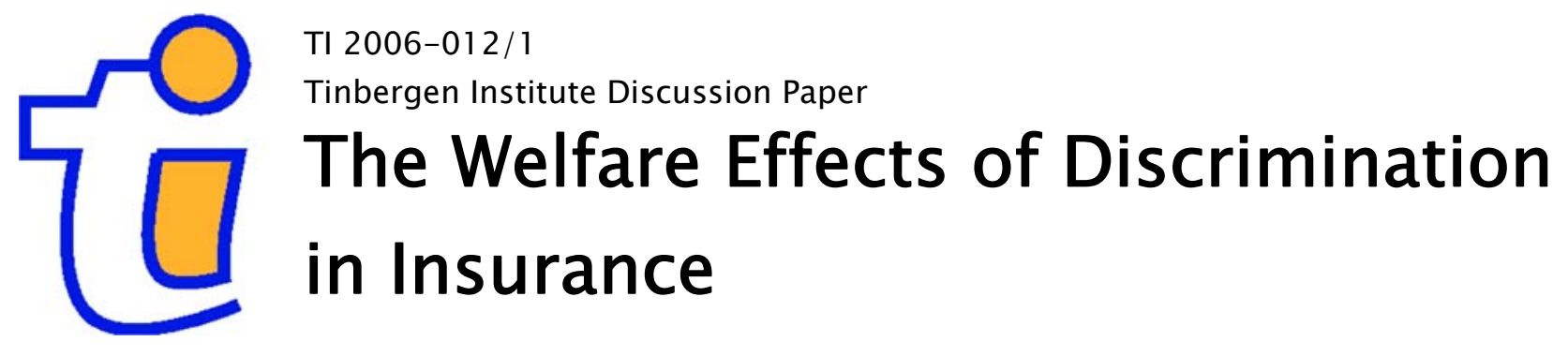

Rob van der Noll

CPB Netherlands Bureau for Economic Policy Analysis, Erasmus University Rotterdam, and Tinbergen Institute. 


\section{Tinbergen Institute}

The Tinbergen Institute is the institute for economic research of the Erasmus Universiteit Rotterdam, Universiteit van Amsterdam, and Vrije Universiteit Amsterdam.

Tinbergen Institute Amsterdam

Roetersstraat 31

1018 WB Amsterdam

The Netherlands

Tel.: $\quad+31(0) 205513500$

Fax: $\quad+31(0) 205513555$

Tinbergen Institute Rotterdam

Burg. Oudlaan 50

3062 PA Rotterdam

The Netherlands

Tel.: $\quad+31(0) 104088900$

Fax: $\quad+31(0) 104089031$

Please send questions and/or remarks of nonscientific nature to driessen@tinbergen.nl.

Most TI discussion papers can be downloaded at http://www.tinbergen.nl. 


\title{
The Welfare Effects of Discrimination in Insurance
}

\author{
Rob van der Noll*
}

\section{CPB Netherlands Bureau for Economic Policy Analysis and Tinbergen Institute, Erasmus University Rotterdam.}

December 29, 2005

\begin{abstract}
We study an insurance model characterized by a continuum of risk types, private information and a competitive supply side. We use the model to investigate the welfare effects of discrimination (also known as risk selection). We postulate that a test is available that determines whether an applicant's risk exceeds a treshold. Excluding the highest risks softens adverse selection, but constitutes a welfare loss for the high risks. In contrast to a lemons market intuition, we find that aggregate surplus decreases when risk aversion is high. When risk aversion is low however, discrimination increases aggregate surplus.
\end{abstract}

JEL Classification: D82, K29.

Keywords: insurance, adverse selection, risk selection, discrimination.

${ }^{*}$ E-mail: vandernoll@few.eur.nl. I thank my advisor Maarten Janssen for invaluable support. Also, I thank Marco Haan and the audience of the CPB Seminar for comments and Francesco Paolucci from iBMG, who was closely involved when this project started. 


\section{Introduction}

When purchasing life insurance, insurees are often required to do a medical test, the result determining at what terms one can be insured, if at all. There is ungoing debate about insurers' information use, for example about genetic discrimination and risk selection (Lemmens 2003). Insurers usually motivate information use by stating that it is a necessary underwriting strategy, since they suffer from adverse selection: insurees have private information about their risk profile and base their decision to buy insurance on this information. Consequently, in a heterogeneous population only the high-risks will purchase insurance. Even though adverse selection is mitigated by this underwriting, the loss of insurance coverage for the high-risks forms a welfare loss. We develop a model of adverse selection in which the two opposing effects are incorporated. Insurers may obtain information about an applicant's risk profile by using some screening technology. We postulate that if the applicant possesses a certain trait (e.g. a gene mutation or virus), his or her risk profile exceeds some upperbound. The objective is to compare welfare in an economy where risk profiles above this upperbound are rejected with an economy in which this is not the case.

We address this question in a setting that has not been studied before. One of the main novelties of our model is that we use a continuum of risk types. The advantage of such a realistic setup is that asymmetric information remains after excluding some of the highest risks. Furthermore, it provides us with a continuous relation between the demand for insurance and important market variables, such as risk aversion. The equilibrium we find has more risk types than contracts. This is not the case in the existing contributions where individuals come in a discrete number of known risk profiles, usually two. In a two-type model, exluding one risk type would imply full information about the remaining pool and the welfare effect of discrimination would be trivial: it would implement the full-information outcome. Our setting permits the important mechanism that when the highest risks are excluded, the expected risk in the pool decreases but individual risks remain unknown. It follows that the actuarially fair premium decreases as well. This in turn gives rise to the following three effects on aggregate surplus: (i) the fall in premium paid due to the overall lower risk in the economy, (ii) some low 
risks will switch to purchasing insurance (softening adverse selection) and (iii) loss of insurance for the high risk types. Our analysis fully balances these three effects and thus shows when total welfare improves due to discrimination and when this is not the case. We thereby contribute to the debate on the desirability of information usage by insurers.

Since discrimination is a mechanism that potentially softens adverse selection, we want to look at adverse selection in isolation from other solutions, like offering an incentive compatible menu-of-contracts where different types choose different combinations of premium and deductible. Additionally, we want to portray a market where there are more risk types than contracts as clearly as possible, and therefore we assume that insurers compete only on the price dimension.

Our results are as follows. The loss of insurance effect (iii) depends on the treshold, which in turn is determined by the nature of the peculiarity tested for and the test technology. For a given treshold, the total effect on welfare depends therefore on the magnitude of effect (i) and (ii) mentioned above. How large will the premium decrease be and how many low risks will be persuaded to buy insurance as a result? The answer depends on the risk attitude in the population. It turns out that when risk aversion is low, the price elasticity of the demarcating low risk consumer is high and many low-risks are gained. As a result, total welfare increases in that case. When risk aversion is very low, a market does not emerge if insurers do not discriminate, while it always emerges in the discrimination regime. On the contrary, when risk aversion is high, even the low risks already have a relatively high willingness to purchase insurance and not many are gained by a premium decrease. Hence, the welfare effect of discrimination is negative in that case.

It is interesting to compare our result with a lemons market model, initiated by Akerlof (1970). In our model, the volume of transactions decreases as a result of discrimination, when risk aversion is high. However, in the lemons model an increase in the lowerbound of the quality distribution always increases the volume of transactions. ${ }^{1}$

\footnotetext{
${ }^{1}$ Suppose that quality is distributed uniformly on $[\underline{\theta}, \bar{\theta}]$ and that buyers are willing to pay $v . E(\theta)$ and that a seller is willing to trade only if the price exceeds his/her quality. Then it
} 
The literature on adverse selection (in insurance) provides the background for the motivation of this paper. ${ }^{2}$ There is quite some literature on discrimination and risk selection. Lemmens (2003) gives an overview of policy and legal issues of genetic discrimination in Canada. The relevance of risk selection in its many forms is discussed by Eggleston (2000), it is furthermore suggested that both demandside cost sharing and risk selection are ways to discourage over-utilization in health insurance. The optimal combination of the two is being analyzed.

A concern raised by insurers is the discrepancy between premium paid and expected costs, while on the contrary others stress the role of solidarity in insurance: low risks should pay more than their expected costs to subsidize the high risks. According to the first concern, a better identification of risk type can be seen as a reduction of discrimination since the discrepancy between premium paid and expected costs decreases. Hoy and Lambert (2000) analyze this effect for genetic screening and assert that the possibility of misclassification is a counterforce.

In contrast to the above papers, we focus on the simple question whether risk selection increases welfare. Our contribution captures the effect that by excluding some risks, the price for the remaining pool decreases, softening adverse selection. We provide clear-cut conclusions about aggregate surplus.

A related common underwriting strategy is risk classification. The difference with risk selection is that with classification, all risk categories are being offered a contract, whereas with selection the highest risks are simply rejected. We use simulations in which the rejected individuals are offered a risk adjusted alternative. This addition does not change our results.

Crocker and Snow developed a theory of risk classification and review it in Crocker and Snow (2000). No sophisticated tests are used but simple data such as age, gender and domicile. Instead, we allow for insurers to gather information by some costly technology, that is potentially more informative, e.g. genetics. They allow for screening of individuals by menus of contracts that have to be made incentive compatible. In contrast, by precluding screening we isolate the

can be shown that in equilibrium the sellers in $\left[\underline{\theta}, \frac{v}{2-v} \underline{\theta}\right]$ trade and the volume of transactions equals $2 \frac{v-1}{2-v} \underline{\theta}$ and is thus increasing in the lowerbound of quality $\underline{\theta}$.

${ }^{2}$ This literature was initiated by Rothschild and Stiglitz (1976) and is surveyed in Dionne et al. (2000). For empirical evidence on adverse selection, see Puelz and Snow (1994). 
merits of risk classification from such other mechanisms that partly resolve adverse selection. Related to this, our simple modelling setup relates clear-cut welfare implications to the degree of risk aversion, as opposed to their conclusions that are more ambiguous. This is due to the complexity of their separating equilibrium and maximization problems, furthermore they need to implement complicated transfers to attain a welfare improvement.

Insurers may also employ some information technology ex-post to investigate the information that was provided by the insuree himself in the insurance application. Insurance law allows for contestability, i.e. when a claim is filed and falsehood is proven, the contract may become void and the payment of indemnity can be denied. This issue is analyzed in Dixit and Picard (2002) and Van der Noll (2005).

Finally, an issue quite different from risk selection occurs when symmetric information is the starting point and an increase of information available to the insuree may create adverse selection. In Subramanian et al. (1999) consumers may learn more about their own risk by doing a genetic test. Using mortality rates for a known gene mutation, they discuss the consequence of this private information for the expected costs of the insurer.

Section 2 presents the model, followed by Sections 3 on equilibrium and 4 on welfare. Section 5 concludes. The proofs are in the Appendix.

\section{The Model}

Consider an insurance market, where the demand side consists of a continuum of individuals who seek to insure the amount of $R$. Individuals have private information about their probability of loss $a_{i}$ and are strictly risk averse, with constant absolute risk aversion (CARA). We assume that $v(m)=-\exp (-\mathrm{cm})$ represents the utility of income, where $c>0$ denotes the CARA coefficient. If no damage occurs, wealth equals $y_{1}$ and in case of damage it equals $y_{2}$, where $y_{1}-y_{2} \geq R$.

The supply side has many insurance firms competing by setting a price $P$. Insurance companies are risk-neutral, have no administration costs and know that the distribution of $a_{i}$ is uniform with support $[0,1]$. In our main result we will assume full insurance: $y_{1}-y_{2}=R$. 
Consider the contract $P$, for individual $i$ the expected utility if this contract is purchased equals:

$$
a_{i} v\left(y_{2}+R-P\right)+\left(1-a_{i}\right) v\left(y_{1}-P\right),
$$

and if no insurance is purchased, expected utility equals:

$$
a_{i} v\left(y_{2}\right)+\left(1-a_{i}\right) v(y) .
$$

Let:

$$
\begin{aligned}
& D_{1}(P)=v\left(y_{1}\right)-v\left(y_{1}-P\right) \\
& D_{2}(P)=v\left(y_{2}+R-P\right)-v\left(y_{2}\right) .
\end{aligned}
$$

Expression $D_{1}$ denotes the ex-post utility cost of insurance if no accident occurred and $D_{2}$ denotes the ex-post utility gain of insurance in case an accident occurred. From (1) and (2), we can derive that, for type $i$, the utility of taking the contract in excess of the utility of not taking the contract equals:

$$
a_{i} D_{2}(P)-\left(1-a_{i}\right) D_{1}(P) .
$$

This expression is intuitive: if an accident occurs (with probability $a_{i}$ ), the utility gain $D_{2}$ is enjoyed, otherwise the cost $D_{1}$ is incurred. We can rewrite the expression as:

$$
F\left(a_{i}, P\right)=\left(D_{1}(P)+D_{2}(P)\right) a_{i}-D_{1}(P) .
$$

An individual purchases the contract iff $F>0$. Since $F$ is increasing in $a_{i}, F(0)<$ 0 and $F(1)>0$ it follows that there exists $\hat{a}$ such that an individual purchases insurance iff. $a_{i}>\hat{a}$, where

$$
\hat{a}=\frac{D_{1}(P)}{D_{1}(P)+D_{2}(P, R)} .
$$

We now introduce the information technology that allows insurers to exclude the highest risks. We assume that a technology is available that allows an insurer to determine whether an individual has a certain trait that is informative about 
the risk profile (e.g. have a disease or gene mutation). Individuals that are found not to possess this trait are known to have a risk profile that does not exceed a certain treshold, denoted by $a^{h}<1 .^{3}$ Yet, their precise risk profile remains unknown beyond that knowledge. We assert that $a^{h}$ depends on the characteristic that is being tested for and on the accuracy of the test.

From the analysis above it follows that the selection of types that demands insurance is then $\left[\hat{a}, a^{h}\right]$ and the expected risk of an insuree equals $E\left(a_{i}\right)=\frac{\hat{a}+a^{h}}{2}$. The benchmark case of no discrimination corresponds to $a^{h}=1$. In what follows, we first characterize equilibrium and consequently investigate the welfare effects of discrimination, i.e. a regime in which $a^{h}$ is less than but close to 1 . Total welfare is defined as the sum of all actors' utilities.

\section{Equilibrium}

Due to perfect competition on the side of insurers, we require that, in expectation, they break even on the contract $P$ :

$$
\begin{gathered}
P-E\left(a_{i}\right) \cdot R=0 \text { or } \\
f(P) \equiv 2 \frac{P}{R}-\frac{D_{1}(P)}{D_{1}(P)+D_{2}(P)}=a^{h} .
\end{gathered}
$$

Observe that for a market to exist, we must have that in equilibrium, $\hat{a}<a^{h}$. The following defines an insurance market in equilibrium:

Definition Let the discrimination treshold be $a^{h} \in(0,1]$. The premium $P^{*}$ characterizes an insurance market in equilibrium if (i) $f\left(P^{*}\right)=a^{h}$ and (ii) $P^{*}<$ $R$. Consumers in $\left[\hat{a}, a^{h}\right]$ purchase insurance. If no such $P^{*}$ exists, no market emerges.

To verify that this is an equilibrium, note that any firm that deviates by setting a lower premium will make a loss because of (3) and that any firm that sets a higher premium attracts no customers. The optimality of the consumer decision is derived above.

\footnotetext{
${ }^{3}$ Insurers may have learned this through past experience.
} 
We now provide a claim about $f(P)$, that is useful to characterize the equilibrium and to show it is unique. In the Appendix we show that:

Claim $1 f(0)=0, f(R)=1 ; \quad \frac{d f}{d P}(0)>0$ and $\frac{d^{2} f}{d P^{2}}<0$.

The above claim implies that equilibrium is unique: $f$ is concave and if it has two intersections with $a^{h}$ then one is in the point where $P=R$ which cannot be an equilibrium. Note that these claims hold for any concave utility function.

Lemma 2 For any concave utility of income, the insurance market equilibrium is unique. If $a^{h}<1$, a market always emerges.

The claim indicates that the premium increases in the upperbound $a^{h}$. Now since the lowerbound of participating types $\hat{a}$ increases in $P$ (which can easily be inferred from the proof of Claim 1), it follows that lowering $a^{h}$ has two opposing effects on market volume. The first is simply that individuals on the upper end of the distribution will be excluded. The second is that more individuals on the lower end of the risk distribution will demand insurance, because the premium decreases. This effect increases market volume and thus softens adverse selection. This will show to be of importance when assessing the welfare effects.

\section{Welfare Analysis}

The following two results will show to be convenient:

Claim $3 f(P)$ increases in $c$.

Claim 4 We have $\lim _{c \rightarrow 0} \hat{a}=P / R$ and $\lim _{c \rightarrow \infty} \hat{a}=0$.

Claim 3 implies that $P^{*}$ decreases in $c$. The more risk averse people are, the more they are willing to pay for insurance, yet the equilibrium premium decreases. This is because $\hat{a}$ decreases in $c$ (more low risks will buy insurance), and the expected risk profile decreases in $c$ and due to the zero profit condition, this decreases the premium. 
We now continue by investigating the welfare that obtains in this market. Since firms make zero expected profits in equilibrium, we add the utility of all types. Formally:

$$
\int_{0}^{1}\left[a_{i} v\left(y_{2}\right)+\left(1-a_{i}\right) v\left(y_{1}\right)\right] d a_{i}+\int_{\hat{a}}^{a^{h}} F\left(a_{i}, P\right) d a_{i} .
$$

Note that the density function of $a_{i}$ equals 1 and is omitted. Also, the first integral is independent of endogeneous variables and since $F$ is lineair in $a_{i}$ it is easy to see that it equals $\frac{v\left(y_{1}\right)-v\left(y_{2}\right)}{2}$. This part of welfare obtains independent of the market. The second integral can be attributed to the operation of a market for insurance and we denote it by $W$. It can be written as:

$$
W=1 / 2\left(\left(D_{1}+D_{2}\right)\left(a^{h}\right)^{2}+\frac{\left(D_{1}\right)^{2}}{D_{1}+D_{2}}-2 D_{1} a^{h}\right) .
$$

When $a^{h}=1$ we have $W=\frac{1}{2} \frac{\left[D_{2}\right]^{2}}{D_{1}+D_{2}}$. Importantly, note that this part of welfare is only realized when a market emerges. The next proposition characterizes the effect of discrimination, i.e. decreasing $a^{h}$ marginally, on welfare. The case where $R$ is smaller than $y_{1}-y_{2}$ as well as a wider variation of $a^{h}$ will be provided by numerical simulations below.

Proposition 5 Let $y_{1}-y_{2}=R$. There exists $c_{1}$ and $c_{2}, 0<c_{1} \leq c_{2}$, such that the effect of a marginal decrease of $a^{h}$ is as follows: (i) if $c<c_{1}$ then welfare $(W)$ increases and (ii) if $c>c_{2}$, then welfare decreases.

The result weighs the three effects that were mentioned in the introduction and can be explained by looking at market volume. When risk aversion is low $\left(c<c_{1}\right)$, the demand for insurance by low risks is relatively low (high lowerbound $\hat{a})$ and their price elasticity is relatively high. The premium decrease due to a lower $a^{h}$ then induces a large decrease in $\hat{a}$, and hence, a large increase in the market volume.

When risk aversion is high however $\left(c>c_{2}\right)$, the low risks are already more inclined to buy insurance (low lowerbound $\hat{a}$ ) and a premium decrease does not induce a large decrease of $\hat{a}$ and thus, the market volume and welfare decrease 
due to lower $a^{h}$. The important mechanism at work is thus the price sensitivity of the pivot $\hat{a}$ which, in turn, is determined by the risk attitude $c$.

Above we assumed that the excluded risks are lost for this economy. It has been shown that this is plausible in real-world insurance, for example by Eggleston (2000). However, it is also observed in some markets that the high risks are offered an alternative insurance policy (known as risk classification). By using numerical simulation, we next analyze if the above welfare result continues to hold if the excluded risk types are being offered a competitive alternative, geared to their risk profile. The risk types that get this contract are in $\left[a^{h}, 1\right]$ and the premium that breaks even for this contract is $P=R \frac{a^{h}+1}{2} .{ }^{4}$ The welfare generated by this contract is:

$$
W^{h}=\int_{a^{h}}^{1} F\left(a_{i}, R \frac{a^{h}+1}{2}\right) d a_{i},
$$

note that since $F\left(a^{h}, \cdot\right)>0$, the area under $F$ is a square plus a triangle, therefore:

$$
W^{h}=\frac{1}{2}\left(1-a^{h}\right)\left[\left(v\left(y_{1}\right)-v\left(y_{2}\right)\right) a^{h}+2 v\left(y_{1}-P\right)-v\left(y_{2}\right)-v\left(y_{1}\right)\right]
$$

where $P=R \frac{a^{h}+1}{2}$. We computed $W+W^{h}$ in Table $1 .^{5}$ The Table shows that the result continues to hold when high-risks get a contract, there is $20 \%$ coinsurance ( $R$ is smaller than $y_{1}-y_{2}$ ) and the decrease in $a^{h}$ is more than marginal. It nicely illustrates Proposition 5: when the CARA coefficient $c$ is 3.5 or higher, excluding some of the highest risks decreases welfare. However, when risk aversion is lower than 3.5, welfare is maximized for $a^{h}<1$ : the welfare loss for high risks that results from discrimination is in that case more than offset by the welfare gain of the intermediate risks. When $c<2$ a market doesn't emerge at all if insurers do not discriminate, and in that case welfare is maximized when the $80 \%$ lowest and $20 \%$ highest risks obtain separate contracts. Of course, the level of $a^{h}$ that can be set in the market depends on screening technologies and the underlying risk determinants.

\footnotetext{
${ }^{4}$ It can be shown that there is no adverse selection of the consumers $\left[a^{h}, 1\right]:$ all types purchase the contract, formally it must be that $F\left(a^{h}, R \frac{a^{h}+1}{2}\right) \geq 0$.

${ }^{5}$ The algorithms are available upon request.
} 


\begin{tabular}{|r|r|r|r|r|r|}
\hline$c:$ & $a^{h}=0,8$ & $a^{h}=0,85$ & $a^{h}=0,9$ & $a^{h}=0,95$ & $a^{h}=1$ \\
\hline 0,001 & 0,00000001 & 0,00000001 & 0,00000000 & 0,00000000 & 0,00000000 \\
\hline 0,5 & 0,00354953 & 0,00226201 & 0,00114772 & 0,00033047 & 0,00000000 \\
\hline 1 & 0,02013119 & 0,01479152 & 0,00903650 & 0,00337247 & 0,00000000 \\
\hline 1,5 & 0,05354369 & 0,04526396 & 0,03448210 & 0,01983445 & 0,00000000 \\
\hline 2 & 0,09500343 & 0,08807487 & 0,07815561 & 0,06254627 & 0,03148265 \\
\hline 2,5 & 0,13312970 & 0,12974048 & 0,12454256 & 0,11554422 & 0,09754395 \\
\hline 3 & 0,16262105 & 0,16264971 & 0,16233828 & 0,16057401 & 0,15483261 \\
\hline 3,5 & 0,18300086 & 0,18551296 & 0,18891018 & 0,19271931 & 0,19580298 \\
\hline 4 & 0,19571150 & 0,19974196 & 0,20556658 & 0,21315842 & 0,22217796 \\
\hline 4,5 & 0,20255250 & 0,20733971 & 0,21455982 & 0,22450944 & 0,23736602 \\
\hline 5 & 0,20511071 & 0,21011745 & 0,21798604 & 0,22925902 & 0,24448385 \\
\hline 5,5 & 0,20462931 & 0,20950261 & 0,21750835 & 0,22938325 & 0,24596011 \\
\hline 6 & 0,20203231 & 0,20655539 & 0,21436285 & 0,22634866 & 0,24357739 \\
\hline 6,5 & 0,19798910 & 0,20204082 & 0,20944004 & 0,22120994 & 0,23860989 \\
\hline 7 & 0,19297856 & 0,19650271 & 0,20337115 & 0,21471377 & 0,23195704 \\
\hline 7,5 & 0,18734128 & 0,19032454 & 0,19659956 & 0,20738414 & 0,22424970 \\
\hline 8 & 0,18131897 & 0,18377544 & 0,18943484 & 0,19958666 & 0,21592868 \\
\hline
\end{tabular}

Figure 1: $W+W^{h}$ for $y_{1}=1.1, y_{2}=.1, R=0.8$ and different values of $c$ and $a^{h}$.

\section{Concluding remarks}

By setting up a model of adverse selection with a continuum of risk types, we assessed the welfare effects (in terms of aggregate surplus) of risk selection (or discrimination) in insurance. The use of the continuum made the problem nontrivial: after selecting some risks out, uncertainty about individuals' risk remains. It also highlights the role of risk aversion in explaining the demand for insurance. Insurers compete on the price dimension only, allowing us to obtain an equilibrium with more risk types than contracts.

Our results contribute to the debate on the desirability of information use by insurers. Only when risk aversion is low, discrimination can be justified on the basis of aggregate surplus. In fact, in this case it might prevent the market from not emerging at all.

\section{Appendix}

In the Appendix we denote by $L$ the loss $y_{1}-y_{2}$.

Claim $1 f(0)=0, f(R)=1 ; \quad \frac{d f}{d P}(0)>0$ and $\frac{d^{2} f}{d P^{2}}<0$. 
Proof. The first two observations are trivially verified. The derivative equals:

$$
\frac{d f}{d P}=\frac{2}{R}-\frac{D_{1}^{\prime}(P) D_{2}-D_{1} D_{2}^{\prime}(P)}{\left[D_{1}+D_{2}\right]^{2}}
$$

and the derivative in 0 is

$$
\frac{d f}{d P}(0)=\frac{2}{R}-\frac{D_{1}^{\prime}(P)}{D_{2}(P)}=\frac{2}{R}-\frac{v^{\prime}\left(y_{1}\right)}{v\left(y_{2}+R\right)-v\left(y_{2}\right)} .
$$

For the latter to be positive it has to be required that:

$$
2>\frac{v^{\prime}\left(y_{1}\right)}{\frac{v\left(y_{2}+R\right)-v\left(y_{2}\right)}{R}} .
$$

Now note that the denominator is simply the slope of the secant line through $y_{2}$ and $y_{2}+R$ and that $y_{2}+R$ is at most $y_{1}$. From the concavity of $v(\cdot)$ it then follows that $v^{\prime}\left(y_{1}\right)<\frac{v\left(y_{2}+R\right)-v\left(y_{2}\right)}{R}$ and this proves the third observation.

Next, note that:

$\frac{d^{2} f(P)}{d P^{2}}=-\left[\frac{D_{1}^{\prime \prime}(P) D_{2}-D_{1} D_{2}^{\prime \prime}(P)}{\left[D_{1}+D_{2}\right]^{2}}-\frac{2\left[D_{1}^{\prime}(P) D_{2}-D_{1} D_{2}^{\prime}(P)\right]\left[D_{1}^{\prime}(P)+D_{2}^{\prime}(P)\right]}{\left[D_{1}+D_{2}\right]^{3}}\right]$.

Now since $D_{1}>0, D_{2}>0, D_{1}^{\prime}>0, D_{2}^{\prime}<0, D_{1}^{\prime \prime}>0$ and $D_{2}^{\prime \prime}<0$ and the fact that $D_{1}^{\prime}(P)+D_{2}^{\prime}(P)<0$, this derivative is negative.

Claim 3 Let $v(m)=-\exp (-c m) \cdot f(P)$ increases in $c$.

Proof. We have to show that $\hat{a}$ decreases in $c$. Recall that

$$
\hat{a}=\frac{D_{1}(P)}{D_{1}(P)+D_{2}(P)}
$$

and let $D_{i c}^{\prime}=\frac{\partial D_{i}}{\partial c}$ for $i=1,2$. Then we can write:

$$
\frac{\partial \hat{a}}{\partial c}=\frac{D_{1 c}^{\prime} D_{2}-D_{2 c}^{\prime} D_{1}}{\left[D_{1}+D_{2}\right]^{2}}
$$

and for the latter expression to be negative, we require that:

$$
\frac{D_{1 c}^{\prime}}{D_{1}}<\frac{D_{2 c}^{\prime}}{D_{2}} .
$$


For the specified utility function, this becomes:

$$
\begin{aligned}
& \frac{-\left(y_{1}-P\right) \exp \left(-c\left(y_{1}-P\right)\right)+y_{1} \exp \left(-c y_{1}\right)}{\exp \left(-c\left(y_{1}-P\right)\right)-\exp \left(-c y_{1}\right)}< \\
& \frac{-y_{2} \exp \left(-c y_{2}\right)+\left(y_{2}+R-P\right) \exp \left(-c\left(y_{2}+R-P\right)\right)}{\exp \left(-c y_{2}\right)-\exp \left(-c\left(y_{2}+R-P\right)\right)}
\end{aligned}
$$

which is equivalent to:

$$
\begin{gathered}
\frac{-\left(y_{1}-P\right) \exp (c P)+y_{1}}{\exp (c P)-1}<\frac{\left(y_{2}+R-P\right) \exp (-c[R-P])-y_{2}}{1-\exp (-c[R-P])} \Leftrightarrow \\
-\frac{P \exp (c P)}{1-\exp (c P)}-\frac{(R-P) \exp (-c[R-P])}{1-\exp (-c[R-P])}<y_{1}-y_{2} .
\end{gathered}
$$

Now let $x_{1}=-P, x_{2}=R-P$, and bring the exponential terms to the denominator, then we can rewrite the above expression as:

$$
\frac{x_{1}}{\exp \left(c x_{1}\right)-1}-\frac{x_{2}}{\exp \left(c x_{2}\right)-1}<L
$$

Now we will show that (i): $\frac{x}{\exp (c x)-1}$ decreases in $x$ and (ii): check inequality (4) for $x_{1}=-L$ and $x_{2}=R$ :

(i): The derivative equals $\frac{\exp (c x)(1-c x)-1}{[\exp (c x)-1]^{2}}$ and we can demonstrate that $e^{x}(1-x)<$ 1 for $x>0$ : in $x=0$, LHS $=$ RHS and $\frac{d L H S}{d x}=-x e^{x}<0$.

(ii) We have $\frac{-L}{\exp (-c L)-1}-\frac{R}{\exp (c R)-1}<L$, which is equivalent to $-\frac{R}{\exp (c R)-1}<$ $L \frac{\exp (-c L)}{\exp (-c L)-1}$, which, in turn, is equivalent to $\frac{R}{\exp (c R)-1}>\frac{L}{\exp (c L)-1}$. Now since $R \leq L$, and $\frac{x}{\exp (c x)-1}$ decreases in $x$ (shown in (i) above) the inequality holds for all $R<L$. When $R=L$, LHS equals RHS.

Due to step (i), it is enough to find for some $x_{1}<-P$ and $x_{2}>R-P$ a weak inequality of (4). This is done in step (ii).

Claim 4 We have (a) $\lim _{c \rightarrow 0} \hat{a}=P / R$ and (b) $\lim _{c \rightarrow \infty} \hat{a}=0$.

Proof. (a) Write $\hat{a}$ as $1 / y(c)$, where $y(c)=1+D_{2} / D_{1}$. By applying the rule of l'Hopital once we obtain that $D_{2} / D_{1} \rightarrow(R-P) / P$ and hence $y(c) \rightarrow R / P$.

(b) From Claim 3 we know that $\hat{a}$ decreases in $c$, therefore it converges. Suppose for sake of contradiction that it converges to a strictly positive number, $\lim \hat{a}>0$. This implies that there exists small $\epsilon>0$ such that $\lim _{c \rightarrow \infty} F(\epsilon, P)<0$ (a low risk type does not buy insurance when $c \rightarrow \infty)$. Now since $F(0, P)=$ 
$-D_{1}(P)$ and $D_{1}(P) \rightarrow 0$ this is contradicted and the result follows.

Proposition 5 Let $R=L$. There exists $c_{1}$ and $c_{2}, 0<c_{1} \leq c_{2}$, such that the effect of a marginal decrease of $a^{h}$ is as follows: (i) if $c<c_{1}$ then welfare $(W)$ increases and (ii) if $c>c_{2}$, then welfare decreases.

\section{Proof. (i)}

We know that when $a^{h}=1$, a market emerges iff

$$
2<\frac{v^{\prime}\left(y_{2}\right)}{\frac{v\left(y_{1}\right)-v\left(y_{1}-R\right)}{R}}
$$

while a market always emerges when $a^{h}<1$. Hence, when the above inequality is violated, a marginal decrease of $a^{h}$ means that in stead of foreclose, a market emerges, which is a welfare improvement. We will now show that a violation of (5) is equivalent to a low value of $c$. When $R=L,(5)$ reads as $\frac{c L}{1-\exp (-c L)}>2$ and LHS increases in $c L$. To complete the proof of the statement, note that $\lim _{c \rightarrow 0} \frac{c L}{1-\exp (-c L)}=1$.

Recall that $P$ is a function of $a^{h}$ via (3). When $R=L$ we have that $D_{1}+D_{2}$ is independent of endogeneous variables. We have that $W=\frac{1}{2}\left\{\left(D_{1}+D_{2}\right)\left(a^{h}\right)^{2}+\frac{\left(D_{1}\right)^{2}}{D_{1}+D_{2}}-2 D_{1} a^{h}\right\}$ and taking the derivative w.r.t. $a^{h}$ we obtain:

$$
\frac{\partial 2 W}{\partial a^{h}}=\left(D_{1}+D_{2}\right) 2 a^{h}+\frac{1}{\left(D_{1}+D_{2}\right)} 2 D_{1} \frac{\partial D_{1}}{\partial P} \frac{\partial P}{\partial a^{h}}-2\left(\frac{\partial D_{1}}{\partial P} \frac{\partial P}{\partial a^{h}}+D_{1}\right),
$$

now substitute $a^{h}=1$, the expressions for $D_{1}$ and $D_{2}$ and rearrange:

$$
[v(y-L)-v(y-P)]\left\{\frac{v^{\prime}(y-P)}{v(y)-v(y-L)} \frac{\partial P}{\partial a^{h}}-1\right\}
$$

with the specific utility function this becomes:

$$
[\exp (-c(y-P))-\exp (-c(y-L))]\left(\frac{c}{\exp (c(L-P))-\exp (-c P)} \frac{\partial P}{\partial a^{h}}-1\right)
$$

We will now show that the latter equality is positive for $c$ large enough. Observe that the first multiplicand is negative. Now recall that when $c \rightarrow \infty, \widehat{a} \rightarrow 0$ and hence $\frac{\partial P}{\partial a^{h}} \rightarrow \frac{R}{2}$ in that case. We will next investigate the fraction between 
brackets $\frac{c}{\exp (c(L-P))-\exp (-c P)}$.

We have that the derivative has the sign of $[1-c(L-P)] \exp (c(L-P))-$ $(1+c P) \exp (-c P)$ and is therefore negative when:

$$
1-\frac{c L}{1+c P}<\exp (-c L)
$$

Now note that the fraction on the LHS increases in $c$, implying that LHS becomes eventually negative. This demonstrates that the derivative is negative for $c$ large enough. We will now show that

$\lim _{c \rightarrow \infty} \frac{c}{\exp (c(L-P))-\exp (-c P)}=0$. Applying the rule of l'Hopital once we obtain that the limit equals $\lim _{c \rightarrow \infty} \frac{1}{(L-P) \exp (c(L-P))+P \exp (-c P)}=0$. Therefore, the fraction between brackets decreases in $c$ for $c$ large enough and converges to zero and

since $\frac{\partial P}{\partial a^{h}} \rightarrow \frac{R}{2}$ their product becomes less than 1 for $c$ large enough. The above demonstrates that $\partial W / \partial a^{h}>0$ for such $c$ and completes the proof.

\section{References}

[1] Akerlof, G. A., 1970. The Market for 'Lemons': Quality Uncertainty and the Market Mechanism. Quarterly Journal of Economics, 84(3), 488-500.

[2] Crocker, K.J., Snow, A., 2000. The theory of risk classification. In: Dionne, G. (Ed.), The handbook of Insurance. Kluwer Academic Publishers, Dordrecht.

[3] Dionne, G. (Ed.), 2000. The Handbook of Insurance. Kluwer Academic Publishers, Dordrecht.

[4] Dixit, A., Picard, P., 2002. On the Role of Good Faith in Insurance Contracting. Princeton University Economic Theory Working Paper No. 02S2. http://ssrn.com/abstract $=303841$.

[5] Eggleston, K., 2000. Risk Selection and Optimal Health Insurance-provider payment systems. Journal of Risk and Insurance 67-2, p. 173.

[6] Hoy, M., Lambert, P., 2000. Genetic Screening and Price Discrimination in Insurance Markets. The Geneva Papers on Risk and Insurance Theory 25, 103-130. 
[7] Lemmens, T., 2003. Genetics and Insurance Discrimination. Health Law Journal Special Edition.

[8] Puelz, R., Snow, A.,1994. Evidence on adverse selection: Equilibrium and cross-subsidization in the insurance market. Journal of Political Economy 102, 236-257.

[9] Rothschild, M., Stiglitz, J.E.,1976. Equilibrium in competitive insurance markets: An essay on the economics of imperfect information. Quarterly Journal of Economics 90, 629-649.

[10] Subramanian, K, Lemaire, J., Hershey, J. C., Pauly, M. V., Armstrong, K., Asch, D.A., 1999. Estimating Adverse Selection Costs from Genetic Testing for Breast and Ovarian Cancer: The case of Life Insurance. Journal of Risk and Insurance 66-4, 531-50.

[11] van der Noll, R., 2005. The Welfare Effects of Contestability in Insurance. Tinbergen Institute Rotterdam, mimeo. 